\title{
What counts as a decision? Predictors of perceived decision making
}

\author{
GRETCHEN B. CHAPMAN and LAURA Y. NIEDERMAYER \\ Rutgers University, Piscataway, New Jersey
}

\begin{abstract}
We examined lay perceptions of what counts as a decision. Eighty-six subjects read 10 scenarios that described clear decisions (e.g., choosing a graduate school), clear "nondecisions" (e.g., an accidental wrong turn), or ambiguous actions (e.g., eating a donut when on a diet or allowing someone else to make the decision). The subjects rated each scenario as to whether the actor had made a decision and also rated six other attributes. The scenarios were rated as clearly illustrating a decision if the actor engaged in a lot of thought and did not act reflexively. Consideration of consequences and having alternatives were moderately related to decision ratings, whereas having self-control problems and the influence of physiological drives showed little relationship with decision ratings. Thus, lay concepts of decision making differ from decision theory in that thoughtful, intentional behavior is more important in defining a decision than are decision theoretic components, such as alternatives and consequences.
\end{abstract}

Imagine the activities that might make up a typical day. A person might wake up, shower, dress, miss the morning bus, wait for the next bus, have an argument with a co-worker, complete a task requested by her boss, select a cafeteria in which to each lunch, have dessert even though she previously vowed to lose weight, accidentally get off at the wrong bus stop on the way home, and stay up late watching a movie. Which of these activities are the result of decision making?

This example illustrates the difficulty inherent in defining which actions constitute a decision. Are automatic or habitual behaviors, such as daily showering, decisions? Is a deviation from a previous plan (such a as a vow to diet) a decision, or is it instead a lapse in decision making? When behaviors have no plausible alternatives (such as waiting for the next bus), are they nevertheless the result of a decision? Although there is much research on the psychological processes underlying decision making, there is little on what qualifies as a decision.

The present study examined lay people's subjective perceptions of what counts as a decision. That is, we addressed the following question: When do people have the subjective perception that a decision has been made? We examined the characteristics of an action, as well as contextual factors, that may influence the perception that the action was the result of an explicit decision.

Portions of this research were presented at the August 1999 meeting of SPUDM (Subjective Probability, Utility, and Decision Making) in Mannheim, Germany. Support was provided by NSF Grant 97-96042, awarded to the first author. Jon Baron, Noel Brewer, Elliot Coups, and two anonymous reviewers provided helpful comments on an earlier draft. Correspondence concerning this article should be addressed to G. B. Chapman, Rutgers University, Psychology Department, 152 Frelinghuysen Road, Piscataway, NJ 08854-8020(e-mail: gbc@ rci.rutgers. edu).
In his textbook on judgment and decision making, Yates (1990, p. 3) defines a decision as "an action taken with the intention of producing favorable outcomes." According to Baron $(2000$, p. 6) decision making is a "a choice of action —of what to do or not do" resulting from inferences made from alternatives, evidence, and goals. Both definitions indicate that decision making is intentional and goal-oriented. Baron's definition also makes explicit that decisions involve multiple alternatives from which to choose. If these definitions are consistent with lay people's perceptions of decision making, we would expect that actions that are intentional, goal-oriented, and have alternatives would be viewed as decisions, whereas those that are unintentional or reflect automatic processing would not be viewed as decisions.

The determinants of experienced decision making can be contrasted with the characterization of a decision put forward by decision theory (e.g., Edwards, 1992; Keeney $\&$ Raiffa, 1993). Decision theory is an analytical framework that can be applied to a choice or judgment to identify the key components and combine those components to compute the optimal choice. In this framework, the key components of a decision include multiple alternatives for choice, the consequences of each alternative, and the characteristics of these consequences used for evaluating them, such as their likelihood and value. Several specific versions of decision theory identify decision algorithms for specific cases. For example, expected utility theory prescribes that, in decision making under uncertainty, the values of each outcome should be weighted by their probabilities and summed for each alternative. The alternative with the highest sum should then be chosen. ${ }^{1}$

Decision theory is a normative theory that prescribes the optimal method of decision making, which is not necessarily how people actually make decisions. Many psychological theories of decision behavior, however, mirror 
decision theory and incorporate the same components with minor modifications (e.g., prospect theory; Tversky \& Kahneman, 1992)..$^{2}$ If lay perceptions of decision making mirror decision theory or the psychological theories based on it, they should incorporate such components as alternatives and consequences.

Decision theory does not imply that decision makers consciously identify alternatives, note their consequences, and estimate the probability and value of each consequence. That is, descriptive decision theories may account for behavior involving choice whether or not decision makers perceived themselves as making decisions and whether or not their experience of decision making mirrors the process postulated by the theory. In fact, many such theories rely on theoretical constructs that are not at all similar to the way most decision makers would describe their decision process. It is, therefore, not clear whether the factors driving the perception of decision making are factors that are central to psychological or to normative theories of decision making.

\section{Previous Research}

Little previous research has explored subjective perceptions of decision making. Two areas of investigation are relevant to the question of when actions seem more or less like a decision. The first research area concerns the interplay between habits and decisions. The second research area concerns failures in self-control that, according to some views, represent lapses in decision making.

The first relevant area of previous investigation has contrasted decision making that employs deliberative processing with that which involves automated or habitual processing. In a series of studies, Aarts, Verplanken, and van Knippenberg (1997, 1998; Verplanken, Aarts, \& van Knippenberg, 1997; Verplanken, Aarts, van Knippenberg, \& Moonen, 1998; Verplanken, Aarts, van Knippenberg, \& van Knippenberg, 1994) contrasted habitual decision making about travel mode with deliberative decision processes. Subjects were asked to make choices for mode of travel (car, bicycle, train, etc.), and these choices were predicted on the basis of attitudes, intentions, and reported habit for car or bicycle use. Intentions had a strong relationship to behavior only when habit strength was weak. Thus, explicit or intentional decisions were less common in the presence of a strong habit. Instructions to deliberate about the decision, however, increased the role of intention and decreased the role of habit (Verplanken et al., 1998; Verplanken et al., 1994), showing that deliberative processing can override strong habits. Habit strength also was related to elaborateness of the decision process. Subjects with strong habits for car use inspected less information when presented with new scenarios for choice of travel mode. In addition, habit affected the type of information search strategy, so that subjects with a strong habit used more noncompensatory, or heuristic-based, search strategies (Verplanken et al., 1997) and tended to base decisions on a small number of cues. Those lower in habit strength used more cues and tended to give equal weight to all the cues (Aarts et al., 1997, 1998).
This research suggests that explicit decision making involves the use of cost and benefit information to select an action. In contrast, a default rule or habit involves less consideration of costs and benefits and a simpler method of combining information. It is important to note, however, that Verplanken, Aarts, and colleagues do not define habitual processing as the absence of decision making but, rather, as a different decision process. It is nevertheless plausible that habitual decision making may correspond to the subjective perception that an action occurred in the absence of decision making.

The second area of previous research relevant to the present research question is the analysis of self-control lapses. Lapses in self-control include such behaviors as breaking one's diet, getting into a fight against one's better judgment, or turning off the alarm clock and sleeping in. Although some analyses describe self-control lapses as the result of explicit decision making that involves conflict (e.g., Ainslie \& Haslam, 1992; Thaler \& Shefrin, 1981), other analyses (Loewenstein, 1996) propose that self-control lapses occur when decision-making capacity is degraded because of the influence of physiological drives (e.g., exhaustion, hunger, fear, or anger). Thus, lapses in self-control are not the result of explicit decision making (e.g., one does not decide to break one's diet) but, instead, represent the absence of explicit decision making. Physiological drives are theorized to override explicit decision making and to result in lapses of self-control. Is this account consistent with subjective perceptions of decision making? If so, actions that constitute loss of self-control and those that are elicited by drive states should not be perceived as decisions.

\section{Present Study}

The goal of the present study was to examine lay people's perceptions of what qualifies as a decision. The study tested three predictions. Our first prediction was that decisions would be perceived as intentional behaviors. Consequently, behaviors that involved careful thought would receive higher decision ratings than would reflexive actions. This prediction is consistent with the definitions offered by Yates (1990) and Baron (2000) and also with the work of Verplanken, Aarts, and colleagues, who found that habitual or routine behaviors were not governed by intention and involved cognitive processes that differed from those underlying explicit decisions. Our second prediction was that decisions involve two key decision-theoretic components: a choice among multiple alternatives and a consideration of the consequences or outcomes of each alternative. Thus, higher decision ratings should be assigned to actions that have alternatives and that result from consideration of consequences. Our third prediction stems from Loewenstein's (1996) account of self-control lapses. Specifically, we predicted that actions would receive lower decision ratings if they represented self-control lapses or if they were influenced by physiological drives, such as hunger or anger.

To test these predictions, subjects were presented with 10 hypothetical scenarios that described a situation and 
an action, and they rated whether the actor had made a decision. Other ratings of the scenarios were then used to predict decision ratings. Attributes predicted to be positively correlated with decision rating included the actor's consideration of long-term consequences, the presence of plausible alternatives to the action taken, and the amount of thought the actor engaged in. Features predicted to be negatively correlated with decision ratings were reflexive or automatic behavior, demonstration of self-control problems, and the influence of physiological drives.

\section{METHOD}

\section{Subjects}

The study subjects were 86 introductory psychology students, who participated as part of a course requirement.

\section{Procedure}

The subjects completed a questionnaire consisting of 10 scenarios. As is shown in the Appendix, two of these scenarios (selecting a graduate school and selecting a driving route) were designed to illustrate behavior that clearly qualified as decision making. Two other scenarios (jump out of truck path and drive toward wrong street) were written to illustrate behavior that was very unlike decision making. The remaining 6 scenarios described ambiguous cases in which actions, although voluntary, might seem unlike decisions because they involved a lack of alternatives, the presence of strong drives, breaking previous plans, strong habits, expected behavior, or ceding decision making power to someone else.

For each scenario, the subjects rated whether the actor made a decision, using a scale from 1 to 5 . The scale endpoint of 1 was labeled definitely not a decision, the endpoint 5 was labeled definitely a decision, and the midpoint 3 was labeled somewhat resembles a decision. After rating each of the 10 scenarios, the subjects were asked to read through each of the scenarios a second time and to answer six additional questions about each scenario, using a 5-point scale for each question. These questions, as shown in Table 1, inquired about how much thought the actor in the scenario engaged in before acting and whether the actor acted reflexively, had plausible alternatives, thought about the long-term consequences, showed self-control problems, and was influenced by physiological drives. Two versions of the questionnaire counterbalanced the order of the scenarios and the order in which the six attributes were rated for each scenario.

\section{RESULTS}

The mean ratings assigned to each scenario are listed in the Appendix. The two scenarios designed to illustrate clear decisions (selecting a graduate school and selecting a driving route) received the highest decision ratings. The two scenarios predicted to receive the lowest decision ratings (jump out of truck path and drive toward wrong street) received the lowest and the third-lowest ratings. (The scenario in which a friend selects the restaurant was rated second-lowest.)

Several contrasts were performed on the mean ratings to confirm that the scenarios were interpreted as had been expected. The two scenarios designed to illustrate clear decisions received higher mean decision ratings than did the six ambiguous scenarios $[F(1,756)=157$, $p<.0001]$, which in turn received higher decision ratings than did the two scenarios designed to illustrate clear nondecisions $[F(1,756)=164, p<.0001]$. The two scenarios designed to illustrate self-control problems (eating donuts and sex without a condom) received higher ratings of self-control problems $[F(1,419)=682, p<$ $.0001]$, and physiological drive $[F(1,419)=250, p<$ $.0001]$ than did the remaining four ambiguous scenarios. Finally, the scenario about eating the only vegetarian option received lower ratings of alternatives than did the other ambiguous scenarios $[F(1,420)=220, p<.0001]$.

The primary question of interest was how decision ratings were related to the other ratings the subjects supplied for each scenario. We computed correlations separately for each scenario, and Table 1 shows a set of six correlations for each of the 10 scenarios. Each correlation used 86 observations (from the 86 subjects), and item means were used to replace the $0.2 \%$ of the data that was missing. The bottom row of the table shows the mean correlations averaged across the 10 scenarios. Means were computed after subjecting correlation coefficients to a Fischer $Z$ transformation, and the significance of these means was determined by a univariate $t$ test using 10 observations (the 10 scenario correlations).

As is shown in Table 1, the three strongest predictors of decision rating were amount of thought (significant in 5 of the 10 scenarios), acting reflexively (significant in 7 of 10), and the presence of plausible alternatives (significant in 3 of 10). The mean correlation was significant for each of these predictors. As was predicted, scenarios were given higher decision ratings if the actor was

Table 1

Pearson Correlations Between Decision Ratings and the Six Attributes in Each Scenario $(N=86)$

\begin{tabular}{lcccccc}
\hline \multicolumn{1}{c}{ Scenario } & $\begin{array}{c}\text { Amount of } \\
\text { Thought }\end{array}$ & $\begin{array}{c}\text { Act } \\
\text { Reflexively }\end{array}$ & $\begin{array}{c}\text { Plausible } \\
\text { Alternatives }\end{array}$ & $\begin{array}{c}\text { Think About } \\
\text { Consequences }\end{array}$ & $\begin{array}{c}\text { Self-Control } \\
\text { Problems }\end{array}$ & $\begin{array}{c}\text { Physiological } \\
\text { Drive }\end{array}$ \\
\hline Select graduate school & $.24^{*}$ & $-.21^{*}$ & .27 & .01 & $-.25^{*}$ & -.09 \\
Select driving route & .02 & $-.27^{*}$ & .21 & -.13 & $-.23^{*}$ & -.05 \\
Eat donut while on diet & $.27^{*}$ & $-.27^{*}$ & $.25^{*}$ & -.00 & -.05 & -.03 \\
Take prescribed medication & .10 & $-.41^{*}$ & .12 & $.25^{*}$ & .08 & -.12 \\
Eat usual pizza meal & .16 & $-.31 \dagger$ & $.30 \dagger$ & .14 & $-.21^{*}$ & .03 \\
Eat vegetarian option & $.47 \dagger$ & $-.24^{*}$ & .20 & .18 & -.20 & -.11 \\
Sex without condom & .06 & -.21 & .12 & -.04 & .12 & -.05 \\
Jump out of truck path & .17 & $-.23^{*}$ & .05 & $.22^{*}$ & .15 & -.12 \\
Friend chooses restaurant & $.31 \dagger$ & .00 & .17 & .06 & -.09 & .12 \\
Drive toward wrong street & $.30 \dagger$ & -.07 & -.13 & .14 & -.02 & $.23^{*}$ \\
Mean Correlation $\neq$ & $.22 \dagger$ & $-.23 \dagger$ & $.16 \dagger$ & .08 & -.07 & -.02 \\
\hline
\end{tabular}

$* p<.05 . \quad \dagger<<.01 . \quad \ddagger$ Significance based on univariate $t$ test computed over 10 scenarios. 
perceived to have engaged in much thought and to have plausible alternatives. Lower decision ratings were assigned when the actor was perceived to have acted reflexively. Consideration of long-term consequences was predictive in 2 of the 10 scenarios, but the mean correlation was only marginal $(r=.08, p=.07)$.

Ratings of self-control problems were negatively related to decision ratings in three scenarios. We predicted a negative relationship, because self-control problems may indicate a lapse in explicit decision making. The scenarios that illustrated this relationship, however, were not designed to illustrate self-control problems, suggesting that some subjects may have interpreted self-control in a manner other than intended. Correlations for selfcontrol were rather variable across scenarios, and the mean correlation was not significant $(r=-.07, p=.17)$.

Ratings of physiological drive were positively related in only one scenario-the one about driving toward the wrong street. This relationship ran counter to our predictions. We had predicted that ratings of physiological drive would be negatively related to decision ratings and that this relationship would most likely appear in scenarios that described strong drive states (such as the scenarios about eating donuts or using a condom). The mean correlation across all scenarios for physiological drive was not significant $(r=-.02, p=.63)$.

\section{DISCUSSION}

The present study revealed that the best predictors of decision ratings were ratings of the amount of thought the actor had engaged in before acting and ratings of whether the actor behaved reflexively or automatically. Apparently, the key characteristics of a decision, according to these subjects, is that it be a deliberate act that has been well thought out. Actions (or inactions) that are not considered or are an automatic response to a situation are not perceived to be decisions. Thus, the definition of a decision applied by subjects appears to be that a decision is a voluntary, intentional act. This result supports our first hypothesis and corresponds to definitions offered by Baron (2000) and Yates (1990). It is also consistent with the work of Verplanken, Aarts, and colleagues, who found that habitual behavior is not intentional and is little influenced by costs and benefits, suggesting that it does not represent explicit decision making.

The present study also revealed that decision ratings were related to the perceived presence of decision alternatives and were marginally associated with consideration of long-term consequences, providing some support for our second hypothesis. These features are inherent components in decision theory, which analyzes decisions as choices among alternatives, where each alternative is described in terms of its consequences. Although inherent to decision theory, these features are apparently less central to lay people's conception of a decision.

Finally, the study revealed very little evidence that the influence of physiological drives or self-control problems were seen as lapses in decision making, contrary to our third hypothesis. The judged influence of physiological drives was related to decision ratings in 1 scenario, but in the counterpredicted direction. Ratings of selfcontrol problems were predictive in 3 of 10 scenarios but were not significant overall. According to one conception of self-control (Loewenstein, 1996), self-control lapses can occur when the influence of physiological drives leads to a suspension of explicit decision making. Consequently, one might predict that actions perceived to be self-control lapses or those seen as influenced by visceral factors would be given low decision ratings. The subjects' ratings did not confirm this prediction.

Judgments made about hypothetical scenarios may not reflect intuitions experienced during actual decision making. For example, the subjective perception of decision making when one falls prey to temptation and eats a forbidden donut may be quite different from one's explanation for that same behavior in someone else (or in one's own previous behavior). Loewenstein (1996) predicts just such a discrepancy, or empathy gap. Specifically, visceral factors cause a lapse in decision making, but the effects of such "hot states" on decision making are difficult to predict, remember, or explain when one is in a "cool state." Consequently, one may attribute a self-control lapse in someone else to poor decision making but experience the same self-control lapse personally as simply the absence of decision making. Such empathy gaps could explain the fact that ratings of self-control problems and the influence of physiological drives were not related to decision ratings in the present study. A study that assessed perceptions of decision making when subjects were actually engaged in the target behavior would allow for a fuller picture of when lapses in decision making are perceived to occur.

Additional research is needed to assess the lay definition of decision making, using methods other than the rating of hypothetical scenarios, as in the present study. Such methods might include interviewing subjects about past decisions they have made (e.g., Sloan, 1987) or asking lay people to explain what defines a decision. We used the latter method in a small study of 18 graduate and undergraduate students. After listing three examples of decisions and three examples of "nondecisions," subjects were asked for a definition of a decision. The subjects gave free responses, such as "conscious mental process in which an action and its possible results are imagined in advance and compared to other possible alternatives." The responses were coded as to whether they included each of four concepts: careful thought, multiple alternatives, consequences, and preferences or benefits. All the responses were independently coded by both authors, with $90 \%$ interrater agreement. Six of the 18 definitions mentioned careful thought, 11 mentioned multiple alternatives, 4 mentioned consequences, and 3 mentioned preferences or benefits. Three participants did not mention any of these concepts but, instead, described personal control, obligations, or the role of feelings. Thus, the two most frequently mentioned criteria were careful thought and multiple alternatives. These same attributes were two of the three most predictive factors in the pres- 
ent study. Acting reflexively, although predictive in the present study, was not mentioned in these definitions, perhaps because it is negatively related. The definitions offered by the subjects were thus in close agreement with the results from the present study.

Judgments of perceived decision making, such as those collected in the present study, are important because of their potential implications for descriptive theories of decision making. Although decision theories do not necessitate a conscious experience of decision making, it is possible that perceptions of decision making may moderate the validity of descriptive theories. That is, theories of decision making might do a better job of accounting for choice behavior of people who perceive themselves to be making decisions. When people do not view their actions as resulting from decisions, decision theories may provide a less accurate account of those actions. This possibility has not yet been tested. An alternative possibility is that subjective perceptions of decision making are, in fact, unrelated to the validity of descriptive decision theories. Such a result would also be interesting, because it would indicate a potential bias in perceptions of decision making.

The fact that the determinants of perceptions of decision making are not in complete alignment with the key decision-theoretic components indicates that lay perceptions of a psychological phenomenon can differ from the framework adopted by researchers. This failure of subjective experience to mirror psychological theories is also evident in other areas of psychology. For example, in memory research, the subjective perception of remembering often coincides with actual information retrieval, as in explicit memory tests. However, sometimes information retrieval occurs in the absence of subjective perception, as in implicit memory tests (e.g., Dienes \& Perner, 1999), or the perception of remembering occurs in the absence of information retrieval, as in false memory (e.g., Loftus, 1997). Other examples include research on confidence judgments and feeling-of-knowing, which indicates that the subjective perception of accuracy is sometimes at odds with actual accuracy (e.g., Yzerbyt, Lories, \& Dardenne, 1998), and research on blindsight, which illustrates that perception can occur without the subjective experience of perception (Schaerli, Harman, \& Hogben, 1999). As a consequence of such discrepancies, lay understanding of mechanisms of behavior can differ from that espoused by researchers. The research approach used in the present study might be usefully applied to other areas of psychology to elucidate the nature of these differences.

\section{CONCLUSIONS}

Ratings of perceived decision making were most closely related to judgments that the action received a lot of thought and did not represent reflexive behavior. Thus, intentional, planned, thoughtful behavior appears to form the basis of lay conceptions of decision making. Decision-theoretic components, such as having alternatives and considering long-term consequences, were less central to lay perceptions of decision making. Finally, self-control lapses or behaviors elicited by visceral drives were not perceived as involving the absence of decision making.

\section{REFERENCES}

Aarts, H., Verplanken,B., \& Van Knippenberg, A. (1997). Habit and information use in travel mode choices. Acta Psychologica, 96, 1-14.

Aarts, H., Verplanken, B., \& van Knippenberg, A. (1998). Predicting behavior from actions in the past: Repeated decision making or a matter of habit? Journal of Applied Social Psychology, 28, 1355-1374.

Ainslie, G., \& Haslam, N. (1992). Self-control. In G. Loewenstein \& J. Elster (Eds.), Choice over time (pp. 177-209). New York: Russell Sage.

Baron, J. (2000). Thinking and deciding (3rd ed). New York: Cambridge University Press.

Dienes, Z., \& Perner, J. (1999). A theory of implicit and explicit knowledge. Behavioral \& Brain Sciences, 22, 735-808.

EDWARDS, W. (ED.) (1992). Utility theories: Measurement and applications. Boston: Kluwer.

KeEney, R. L., \& RaIfFA, H. (1993). Decisions with multiple objectives: Preferences and tradeoffs. New York: Cambridge University Press.

Loewenstein, G. (1996). Out of control: Visceral influences on behavior. Organizational Behavior \& Human Decision Processes, 65 , 272-299.

Loftus, E. F. (1997). Memories for a past that never was. Current Directions in Psychological Science, 6, 60-65.

Schaerli, H., Harman, A. M., \& Hogben, J. H. (1999). Blindsight in subjects with homonymous visual field defects. Journal of Cognitive Neuroscience, 11, 52-66.

SloAn, T. S. (1987). Deciding: Self-deception in life choices. New York: Methuen.

Thaler, R. H., \& Shefrin, H. M. (1981). An economic theory of selfcontrol. Journal of Political Economy, 89, 392-406.

Tversky, A., \& Kahneman, D. (1992). Advances in prospect theory: Cumulative representations of uncertainty. Journal of Risk \& Uncertainty, 5, 297-323.

Verplanken, B., Aarts, H., \& van Knippenberg, A. (1997). Habit, information acquisition, and the process of making travel mode choices. European Journal of Social Psychology, 27, 539-560.

Verplanken, B., Aarts, H., van Knippenberg, A., \& Moonen, A. (1998). Habit versus planned behavior: A field experiment. British Journal of Social Psychology, 37, 111-128.

Verplanken, B., Aarts, H., van Knippenberg, A., \& van KnippenBERG, C. (1994). Attitude versus general habit: Antecedents of travel mode choice. Journal of Applied Social Psychology, 24, 285-300.

YATES, J. F. (1990). Judgment and decision making. Englewood Cliffs, NJ: Prentice-Hall.

Yzerbyt, V. Y., Lories, G., \& Dardenne, B. (Eds.) (1998). Metacognition: Cognitive and social dimensions. Thousand Oaks, CA: Sage.

ZsAmbOK, C. E., \& KleIN, G. (EDS.) (1997). Naturalistic decision making. Mahwah, NJ: Erlbaum.

\section{NOTES}

1. Decision theory encompasses not only choice, but also judgments, such as ranking or pricing. The present study, however, focuses on choice, both for simplicity and because of its apparent centrality to perceptions of decision making. In a sample of 18 students asked to define a decision, 13 used the word "choice" or "choose."

2. Other psychology theories, such as naturalistic decision making (Zsambok \& Klein, 1997) do not use decision theory as their basis and do not include decision-theoretic components. 


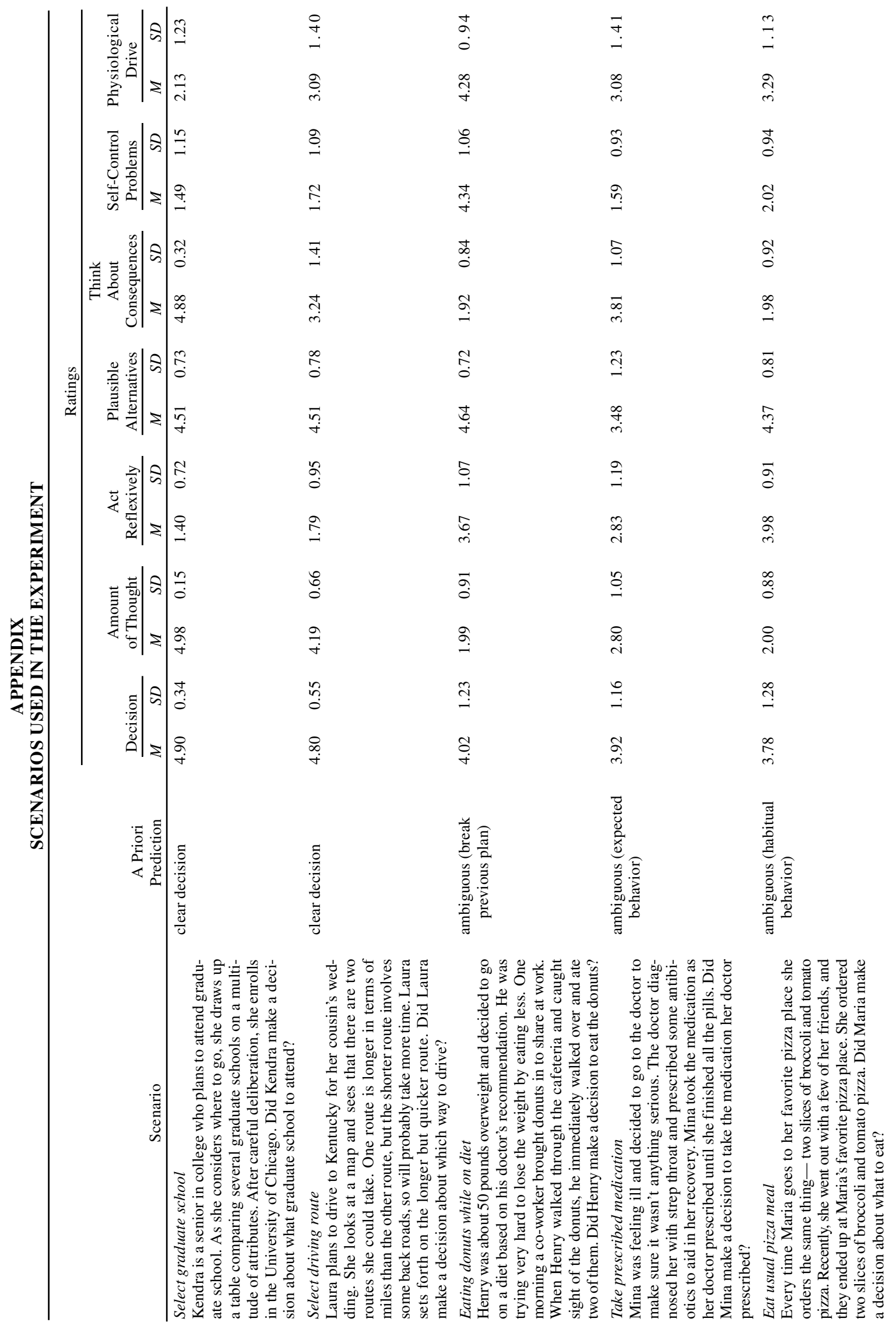




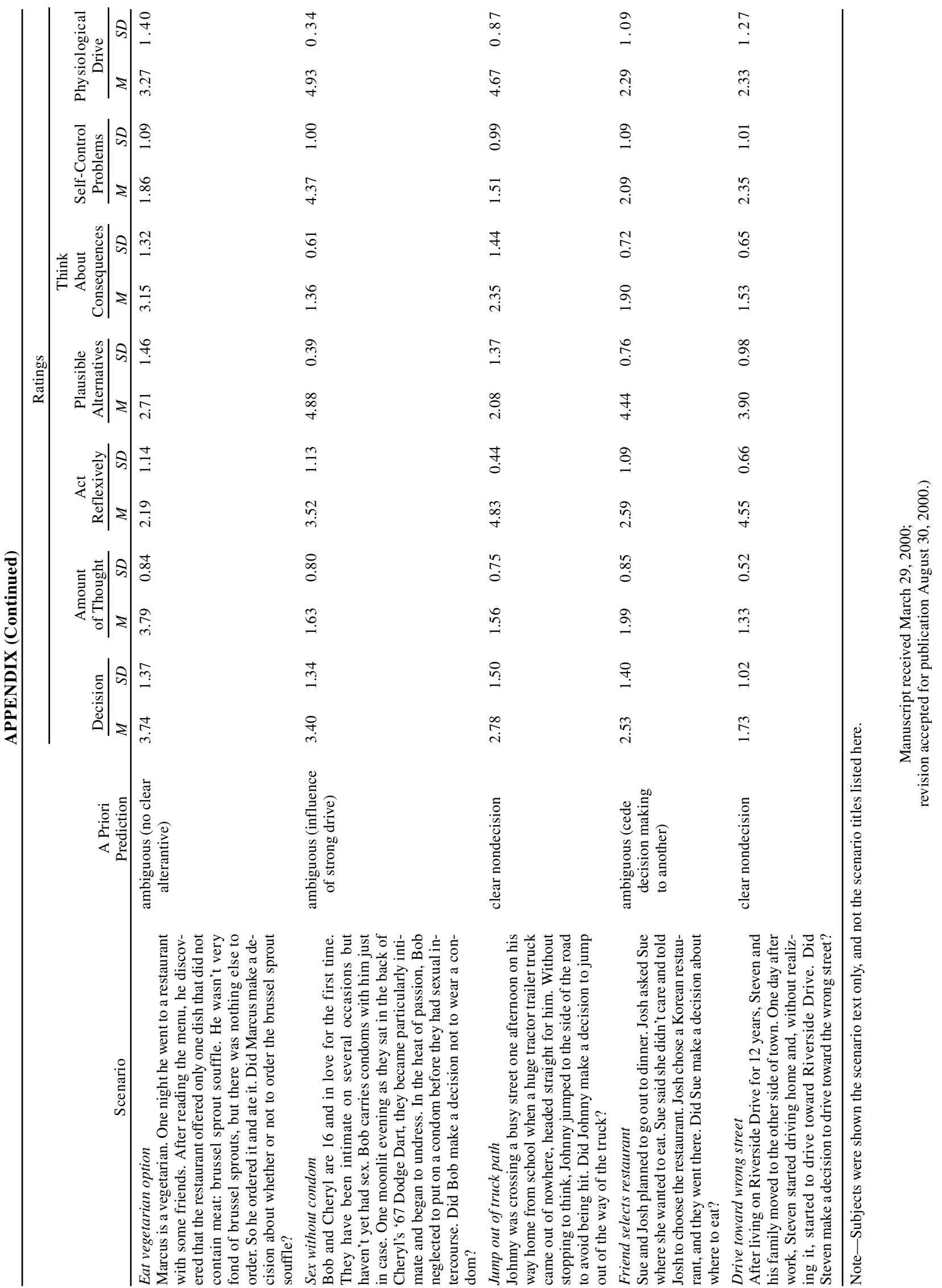

